\title{
Synthesis and in-depth characterization of Ga-based structured catalysts: enhancing glycerol conversion
} \author{
Fiorilli, ${ }^{[\mathrm{c}]}$ Damien P. Debecker, ${ }^{[\mathrm{d}],{ }^{*}}$ and Carmela Aprile ${ }^{\left[\mathrm{a},{ }^{*}\right.}$ \\ [a] Unit of Nanomaterials Chemistry (CNANO), Department of Chemistry, University of Namur, 5000 Namur, Belgium \\ [b] SIAM, Department of Physics, University of Namur, 5000 Namur, Belgium \\ [c] Department of Applied Science and Technology, Politecnico di Torino, Institute of Chemistry, 10129 Torino, Italy \\ [d] Institute of Condensed Matter and Nanosciences - UCLouvain, 1348 Louvain-la-Neuve, Belgium
}

Alvise Vivian, ${ }^{[a]}{ }^{[}$Loraine Soumoy, ${ }^{[a]}$ Luca Fusaro, ${ }^{[a]}$ Pierre Louette, ${ }^{[b]}$ Alexandre Felten, ${ }^{[b]}$ Sonia

\begin{abstract}
Mesoporous Ga-silicates were efficiently prepared using a sustainable and continuous synthesis procedure. The novel solids showed record activity in the synthesis of solketal from glycerol and acetone. Three different Si/Ga ratios of 34,74 and 148 were investigated and all materials displayed favorable features for catalytic applications such as high surface area and calibrated mesoporosity. The insertion of Ga predominantly as single site in tetrahedral coordination in the silica framework was elucidated by XPS, using the Auger parameter of $\mathrm{Ga}$ in a Wagner plot representation. The speciation of $\mathrm{Ga}$ was further clarified using solid state ${ }^{71} \mathrm{Ga}$ NMR spectroscopy, confirming the formation of mainly isolated $\mathrm{Ga}$ species. Consistently, the aerosol-made Ga-silicates displayed outstanding turnover frequencies (up to $677 \mathrm{~h}^{-1}$ ) and selectivity, markedly outcompeting other reference metallosilicate catalysts reported in literature. Moreover, the most active catalyst was successfully reused in multiple catalytic cycles thus proving its stability under the selected reaction conditions.
\end{abstract}

\section{Introduction}

Mesoporous metallosilicate materials are an important class of structured solids possessing high specific surface area, large pore volume and rich surface chemistry. A large number of applications have been proposed in the fields of adsorption, pollutant remediation, sensors, drug delivery and catalysis [1,2]. The importance of silica-based materials is further enhanced by the possible functionalization of the surface as well as by the selective isomorphic substitution of silicon with different elements. Both approaches constitute a key pillar in the preparation of innovative heterogeneous catalysts. For instance, the insertion as singlesite of trivalent cations such as $\mathrm{Ga}^{3+}$ is associated with the generation of Brønsted and Lewis acid sites, useful for a wide range of catalytic reactions [3-5]. The isomorphic substitution of silicon with gallium in various zeolites as well as the synthesis of gallosilicates analogues of faujasite [6] and analcite is well known. The introduction of gallium within the silica architecture resulted in improved catalytic performances in the aromatization of olefins and paraffins [7-9]. Mesoporous gallium silicates have also been exploited for a series of sustainable chemical processes including the synthesis of alkyl lactates and other valuable chemicals from glycerol and its derivatives [10,11]. In particular, it has been already reported that Ga-MCM-41 nanoparticles are one of the most efficient heterogeneous catalysts in the conversion of glycerol into solketal [10].

Recently, the aerosol-assisted sol-gel process emerged as an effective method to produce structured mesoporous silica-based catalysts presenting a metal inserted as single site in the framework [12]. The method implies the atomization of a solution containing molecular precursors which undergo inorganic polycondensation during the rapid drying of the aerosol droplets. By applying this process in the presence of a sacrificial pore-generating agent (e.g. amphiphilic surfactant which self-assemble into micelles under the effect of solvent evaporation) it is possible to design mesostructured particles with a precise control on the texture [13-16]. The advantages of this process combine a reduced waste generation, a limited number of steps, and low environmental impact [17]. In addition, the process for the preparation of catalyst can be easily scaled up and can be run in a continuous mode, making it attractive from an industrial perspective [12, 18]. The aerosol processes have already found application in the production of several metallosilicates displaying excellent catalytic performance in numerous chemical reactions. A few examples are the production of SiAl oxides for hydrocarbon cracking, SiW and SiMoAl oxides for the metathesis of olefin, SiTi oxides for selective oxidation and recently the synthesis of surface functionalized metallosilicate catalysts [13, 19-25]. To the best of our knowledge, the synthesis of mesoporous Ga-silicates via aerosol process has never been reported.

In the synthesis of metallosilicate catalysts, a central pillar is represented by the investigation of the coordination number/geometry of the metal centers inserted as single site within the silica matrix. Describing and quantifying the different species is of fundamental importance since it is strongly correlated with the catalytic performance. In the case of gallium silicates, an in-depth characterization of the coordination environment of gallium is rarely reported in literature. The low gallium concentration in the solids, frequently surrounded by an amorphous environment, makes the characterization of these materials rather challenging. Solid state NMR spectroscopy of ${ }^{71 / 69} \mathrm{Ga}$ and X-ray photoelectron spectroscopy (XPS) are two powerful characterization techniques that can be embraced to elucidate the nature of $\mathrm{Ga}$ environment. The possibility to use solid state ${ }^{69 / 71} \mathrm{Ga}$ NMR has been reported in few studies involving crystalline zeolitic gallosilicates and Ga-MCM-41 solids with the aim of both detecting and distinguishing the contribution related to tetrahedrally and octahedrally coordinated gallium atoms in these structures [26-29]. However, solid state gallium NMR remains challenging due to the quadrupolar nature of both isotopes that are affected by quadrupolar broadening. Despite this, we were able to measure even lower Si/Ga ratios compared to the previously reported studies, working under high magnetic field and using high magic angle spinning (MAS) frequencies to reduce the chemical shift anisotropy effects [30]. For the same purpose, XPS and chemical speciation plots have been extensively employed as useful tools to determine the degree of metal insertion in silica-based solids. As an example, the use of XPS via the Auger parameter in a 
Wagner plot representation, allowed to separate contributions related to intra- and extra-structural zinc species within the same Zn-MCM-41 sample [31]. In spite of this, only few such XPS studies have considered gallium and in particular only two examples describing the use of a Wagner plot for gallium compounds have been reported in the literature until now [32, 33].

In the present work, a deep structural investigation of Ga-silicates prepared for the first time using the aerosol assisted process provides strong evidences of the relation between the nature of Ga species and their catalytic activity. The insertion of gallium as single site in the silica structure was demonstrated via solid state ${ }^{71} \mathrm{Ga}$ MAS NMR and XPS using the Auger parameter. These materials exhibited high performance in the acetalization of glycerol with acetone to produce solketal, outcompeting other reference catalysts reported in literature. This reaction is of particular relevance in the context of sustainable production of useful bio-based chemicals [34].

\section{Experimental}

\subsection{Characterization}

Transmission electron microscopy (TEM) micrographs were collected on a Philips Tecnai 10 microscope using an acceleration voltage of $80 \mathrm{kV}$. The amount of metal (gallium) in the catalysts was obtained by inductively coupled plasma optical emission spectroscopy (Optima 8000 ICP-OES Spectrometer). The samples were prepared digesting $10 \mathrm{mg}$ of the material in a mixture of $100 \mu \mathrm{L}$ of aqua regia and $600 \mu \mathrm{L}$ of HF $48 \%$. Nitrogen adsorption-desorption isotherms were acquired at $77 \mathrm{~K}$ with a Micromeritics Tristar 3000 volumetric adsorption analyzer. The samples were outgassed under reduce pressure at $150^{\circ} \mathrm{C}$ overnight before the analysis. To calculate the specific surface area, the Brunauer-Emmet-Teller (BET) method was applied to the nitrogen adsorption-desorption isotherms in the $0.05-0.20$ relative pressure range. The mean pore diameter was determined from the adsorption branches of the isotherm using the Barrett-Joyner-Halenda $(B J H)$ method. The total pore volume $\left(V_{t}\right)$ was derived from the quantity adsorbed at a relative pressure of 0.99 .

${ }^{71} \mathrm{Ga}$ solid state NMR magic angle spinning (MAS) spectra were recorded at room temperature on a Bruker Avance-500 spectrometer operating at $11.7 \mathrm{~T}$ using a $2.5 \mathrm{~mm}$ Bruker CP-MAS probe. The solids were prepared in a $2.5 \mathrm{~mm}$ zirconia rotor and analyzed with a spinning frequency of $25000 \mathrm{~Hz}$. The spectra were recorded using an Hahn echo sequence and the following acquisition parameters: a relaxation time of $0.3 \mathrm{~s}$, an excitation of $2.6 \mu \mathrm{s}\left(90^{\circ}\right)$ and an acquisition time of 16 ms. Data processing includes the multiplication of the FID (Free Induction Decay) by a line broadening factor of $1000 \mathrm{~Hz}$, zero-filling, Fourier transformation and phase and baseline corrections. Fourier transformation from the echo maximum ensured a flat baseline with only zero-order phase correction. The chemical shift scale was calibrated at room temperature using $\mathrm{Ga}\left(\mathrm{NO}_{3}\right)_{3}$ as the reference compound $(0.0 \mathrm{ppm})$.

X-ray photoelectron spectroscopy characterizations were carried out on a ThermoFisher ESCALAB 250Xi spectrometer, using a monochromatic Al Ka X-ray source (1486.6 eV) and a hemispherical deflector analyzer (SDA) working at constant pass energy (CAE). The diameter of the X-ray spot was set to $300 \mu \mathrm{m}$. The base pressure of the analyzer chamber was amounted to $2 \cdot 10^{-8}$ $\mathrm{Pa}$. A flood gun, using electrons and Ar ions, was used to compensate for charges induced by photoemission. Survey spectra were recorded with a pass energy of $150 \mathrm{eV}$ that was decreased to $25 \mathrm{eV}$ for high-resolution spectra. Chemical components on photoemission spectra as well as Auger signals were fitted with Gaussian-Lorentzian lineshape. The Gaussian-Lorentzian (L/G) ratio, FullWidth at Half Maximum

(FWHM), kinetic energy and intensity (peak area) were adjusted during the procedure.

Brønsted and Lewis acidity of the sample Ga-74 were investigated by Fourier Transform Infrared Spectroscopy (FT-IR) of ammonia adsorption. FT-IR measurements were performed in transmission mode by using a Bruker Tensor 27 spectrometer equipped with a liquid nitrogen-cooled mercury-cadmium-telluride (MCT) detector, operating at a $2 \mathrm{~cm}^{-1}$ resolution. A pretreatment was carried out using a standard vacuum frame, in an IR cell equipped with $\mathrm{KBr}$ windows. The sample was pressed into self-standing wafers and outgassed $1 \mathrm{~h}$ at $400^{\circ} \mathrm{C}$ before adsorption of ammonia at room temperature.

Adsorption of ammonia was studied in the pressure range $0.01-20.0$ mbar: the reversible fraction of the adsorbed ammonia was then removed by prolonged outgassing at room temperature.

\subsection{Synthesis of Ga-silicates}

Solution A was prepared by pre-hydrolysis of TEOS $\left(97 \% ; 5.5 \cdot 10^{-3} \mathrm{~mol} ; 12 \mathrm{~g}, \mathrm{TCl}\right)$ in $\mathrm{HCl} 0,01 \mathrm{M}(0,6 \mathrm{~mol} ; 20 \mathrm{~g}$; pH 2). Solution B was prepared by solvating the structuring agents $\left(0.68 \cdot 10^{-3} \mathrm{~mol} ; 3.9 \mathrm{~g}\right.$ Pluronic P123, Sigma Aldrich) in absolute ethanol (45 $\left.\mathrm{g}\right)$ and $\mathrm{HCl} 0.01 \mathrm{M}(0.22 \mathrm{~mol} ; 8 \mathrm{~g} ; \mathrm{pH}$ 2). Solutions $\mathrm{A}$ and $\mathrm{B}$ were stirred $(300 \mathrm{rpm})$ at room temperature for 40 minutes and 15 hours, respectively. The gallium precursor $\left(\mathrm{Ga}\left(\mathrm{NO}_{3}\right)_{3} \cdot \mathrm{xH}_{2} \mathrm{O}, \mathrm{ROTH}\right)$ was then added to solution $\mathrm{A}$ at the desired $\mathrm{Si} / \mathrm{Ga}$ molar ratio. When the metal precursor was dissolved, solution $\mathrm{B}$ was added to solution $\mathrm{A}$ and the mixture was stirred for 30 minutes ( $300 \mathrm{rpm}$ ). The sol was then atomized ( 2 bar) using a TSI 6-Jet 9306A atomizer, resulting in aerosol droplets that were dried by passing through a quartz tube heated to $350^{\circ} \mathrm{C}$. The obtained powder was collected on a cellulose filter $(0.45 \mu \mathrm{m})$, dried overnight at $80^{\circ} \mathrm{C}$ and then calcined in air at $550^{\circ} \mathrm{C}$ for 5 hours (ramp: $\left.1^{\circ} \mathrm{C} / \mathrm{min}\right)$.

\subsection{Synthesis of Ga-impregnated}

A gallium nitrate solution was prepared by adding $500 \mu \mathrm{L}$ of $0.02 \mathrm{M} \mathrm{HCl}$ and then $1 \mathrm{~mL}$ of absolute ethanol to $\mathrm{Ga}\left(\mathrm{NO}_{3}\right)_{3} \cdot \mathrm{xH}_{2} \mathrm{O}$ ( $0.025 \mathrm{~g} ; \mathrm{Si} / \mathrm{Ga}=37)$. In a $10 \mathrm{~mL}$ beaker, $150 \mathrm{mg}$ of a silica-based aerosol solid was impregnated, drop by drop, with the gallium nitrate solution. The catalyst was then dried in an oven at $65^{\circ} \mathrm{C}$ for two hours, and then calcined in air at $550^{\circ} \mathrm{C}$ for 5 hours. 


\subsection{Catalytic tests}

Acetalyzation of glycerol with acetone: in a round bottom flask, $10 \mathrm{mg}$ of the selected catalyst was added in a mixture containing glycerol $(99 \%, 0.01 \mathrm{~mol}, 0.92 \mathrm{~g})$ and acetone $(0.04 \mathrm{~mol}, 2.32 \mathrm{~g})$. The reaction blend was then vigorously stirred $(800 \mathrm{rpm})$ at $50^{\circ} \mathrm{C}$ during a selected time. At the end of the reaction, absolute ethanol $(3 \mathrm{~mL})$ was added to the reaction media. The catalyst was then isolated by centrifugation (4500 rpm, $10 \mathrm{~min}$ ) and the supernatant was analyzed by ${ }^{1} \mathrm{H}-\mathrm{NMR}$ with DMSO as deuterated solvent.

Leaching tests: in a round bottom flask, $25 \mathrm{mg}$ of the selected catalyst was added in a mixture containing glycerol $(99 \%, 0.01 \mathrm{~mol}$, $0.92 \mathrm{~g})$, absolute ethanol $(0.70 \mathrm{~mL})$ and acetone $(0.04 \mathrm{~mol}, 2.32 \mathrm{~g})$. The reaction blend was then vigorously stirred $(800 \mathrm{rpm})$ at $50^{\circ} \mathrm{C}$ during $1 \mathrm{~h}$. After this time, the catalyst was isolated by hot filtration $\left(\sim 50^{\circ} \mathrm{C}\right)$. The filtrate was then centrifuged $(4500 \mathrm{rpm}, 5$ $\min$ ) and $500 \mu \mathrm{L}$ of the supernatant was analyzed by ${ }^{1} \mathrm{H}$-NMR with DMSO as deuterated solvent. The filtrate was kept at $50{ }^{\circ} \mathrm{C}$ for another $5 \mathrm{~h}$ under $800 \mathrm{rpm}$ stirring. Another NMR analysis of the reaction mixture was then performed.

Recycling tests: in a round bottom flask, $50 \mathrm{mg}$ of Ga-74 was added in a mixture containing glycerol $(99 \%, 0.017 \mathrm{~mol}, 1.56 \mathrm{~g})$ and acetone $(0.068 \mathrm{~mol}, 3.95 \mathrm{~g})$. The reaction blend was then vigorously stirred $(800 \mathrm{rpm})$ at $50^{\circ} \mathrm{C}$ during $1 \mathrm{~h}$. At the end of the reaction, absolute ethanol $(3 \mathrm{~mL})$ was added to the reaction media. The catalyst was then recovered by centrifugation ( $4500 \mathrm{rpm}$, $10 \mathrm{~min}$ ) and the supernatant was analyzed by ${ }^{1} \mathrm{H}-\mathrm{NMR}$ with DMSO as deuterated solvent. The catalyst was washed three times with acetonitrile and calcined in air at $450^{\circ} \mathrm{C}$ for $2 \mathrm{~h}\left(\right.$ rate: $\left.2^{\circ} \mathrm{C} \mathrm{min}-1\right)$. The subsequent catalytic tests were carried out by repeating this procedure and by adapting the reactants in function of the mass of the recovered catalyst.

\section{Results and Discussion}

The aerosol-assisted sol-gel process was employed to synthesize three different Ga-silicates, bearing respectively a nominal silicon to gallium ratio of 37 (Ga-37), 74 (Ga-74) and 148 (Ga-148). All the materials were characterized using several techniques, inter alia, transmission electron microscopy (TEM), X-ray photoelectron spectroscopy and ${ }^{71}$ Ga solid-state NMR spectroscopy, $\mathrm{N}_{2}$ physisorption, and ICP-OES.

All solids consist of spherical particles originating from the very fast drying of the atomized aerosol droplets (Figure 1). The thermal removal of $\mathrm{P} 123$, used as templating agent, allows obtaining well visible "wormlike" mesopores of 6-7 nm in the core together with lamellar domains in the external part of the particles. The Ga loading was quantified by inductively coupled plasma optical emission spectroscopy (ICP-OES) and the resulting $\mathrm{Si} / \mathrm{Ga}$ ratios for all the materials were in good agreement with the nominal composition (Table 1). An in-depth characterization to assess the coordination environment of the Ga metal centers present in the silica matrix is of fundamental importance. In this work, the chemical environment of gallium for all the synthesized solids was elucidated using both X-ray photoelectron spectroscopy (XPS) and solid-state ${ }^{71} \mathrm{Ga}$ NMR.

The XPS results for the solid Ga-148 are not presented due to the too low Si/Ga ratio, close to the lower limit of sensitivity. Hydrated gallium oxide $\left(\mathrm{Ga}_{2} \mathrm{O}_{3}\right)$ was chosen as the reference compound and an additional material called "Ga-impregnated" was synthesized taking inspiration from Liu et al [35] to obtain extra-framework gallium species. This material was synthesized by impregnating an aerosol material consisting of pure silica with a gallium nitrate solution to obtain a theoretical $\mathrm{Si} / \mathrm{Ga}$ ratio of 37 (see experimental section).

As expected, the XPS analysis of standard gallium levels $2 p$ and $3 d$ in $\mathrm{Ga}-37, \mathrm{Ga}-74$ and $\mathrm{Ga}_{2} \mathrm{O}_{3}$ solids did not allow to differentiate significantly the energy contributions associated with the different Ga species (see Figure S1). In the literature, only few studies address the determination of the chemical state of gallium by XPS [32, 33]. The standard use of only chemical shifts of binding energies can be in some cases limiting and for this reason, to better discriminate different energy contributions the use of the Auger parameters is worth to be considered. The Auger parameter has been used for multiple elements and its usefulness is even better exploited when the data are presented in a graphical plot known as Wagner plots. An example is represented by the study conducted by Bourque et al.[32], where a Wagner plot was used as a tool to differentiate the chemical state of gallium in galliumcryptand complexes. In addition, Collard et al.[31] showed that it was possible to distinguish, using the Wagner plot, contributions related to zinc as an isomorphic substituent of silicon atoms and extra-structural zinc within the same Zn-MCM-41 sample.

The graphical representation of the most intense photoelectron line binding energies (abscissa) versus the kinetic energy position of the sharpest Auger line (ordinate) is known as a Wagner plot.

The Auger parameter is the intercept of the linear relationship in equation (1):

$$
E_{K}=\alpha^{\prime}-E_{B}
$$
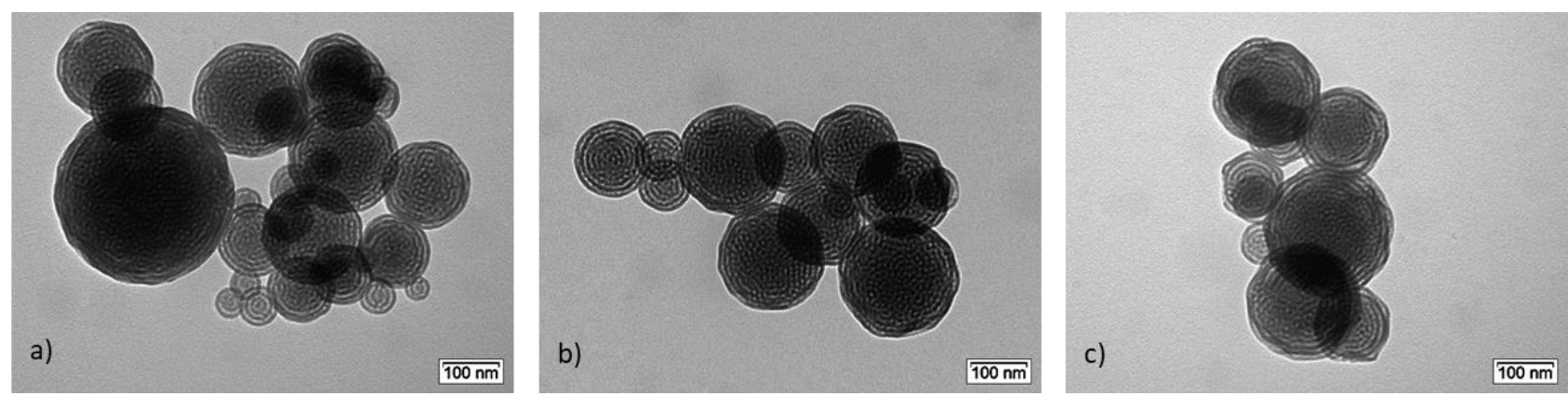

Figure 1. TEM micrographs of Ga-37 (a), Ga-74 (b) and Ga-148 (c) calcined at $550^{\circ} \mathrm{C}$ 
where $E_{K}$ is the kinetic energy of the Auger electron, $\alpha^{\prime}$ the modified Auger parameter and $E_{B}$ is the binding energy of Ga3 $d_{5 / 2}$. This relation can be represented in a Wagner plot on straight lines with slope 1. Moreover, it is possible to show that the relation between $E_{K}$ and $E_{B}$ in a Wagner plot can be expressed by the equation (2) [36, 37]:

$$
\begin{aligned}
& E_{K}=\left[\text { const }+2\left(V_{M}+k Q\right)\right]-3 E_{B} \\
& I=\left[\text { const }+2\left(V_{M}+k Q\right)\right]
\end{aligned}
$$

The value [const $\left.+2\left(V_{M}+k Q\right)\right]$ is related to the initial state of the ionized atom, where $V_{M}$ is the local Madelung potential, $Q$ is the ground-state valence charge and $\mathrm{k}$ is the change in core potential resulting from the removal of a valence electron [37]. Equation (2) shows that compounds with similar initial state effects will appear in a Wagner plot on a straight line with slope 3.

Initial state effects can be seen as shifts in the orbital energies of an atom before it is subject X-ray irradiation. These effects are generally considered to represent the "chemical shift" as a result of ground state electronic structure and are a function of the valence structure of the core atom. These shifts are related to the electronic states and structural parameters of the bonded atoms. The Auger parameter and the Wagner plot representation has been used for multiple elements to study the dependence of the local electronic structure on the atomic environments. An example is represented by the study conducted by Bourque et al.[32], where a Wagner plot was used as a tool to differentiate the chemical state of gallium in gallium-cryptand complexes. In addition, Collard et al. [31]showed that it was possible to distinguish, using the Wagner plot, contributions related to zinc as an isomorphic substituent of silicon atoms and extra-structural zinc within the same Zn-MCM-41 sample.

The Wagner plot presented in Figure 2 shows the graphical representation of the most intense photoelectron line binding energies of $3 d_{5 / 2}$ photoelectrons ( $x$ ) versus the kinetic energy of the most intense core-core-core Auger line (y) for Ga-37, Ga-74, Gaimpregnated and bulk $\mathrm{Ga}_{2} \mathrm{O}_{3}$. On the Wagner plot, the modified Auger parameters are represented by the plain lines with a slope of 1 and the initial state effects I is represented by the dashed lines with a slope of 3 .

According to the study conducted by Wagner and Bourque et al. on the elemental Auger parameter and chemical state plots[32, 38], compounds present on a line with a slope of 3 usually show similar initial state properties (before the interaction with a photon) of the element inside the compound, while in the case of compounds gathered on a line with a slope of 1 (identical modified Auger parameters) similar final state effects are expected (after the interaction with a photon). In Figure 2, all the studied Ga-containing solids are sitting on the same line with a slope of 3 but on different lines with slopes of 1, which suggest that they present differences that can be explained mostly in terms of final state effects[32, 39]. These differences could be attributed to various characteristics such as the particle size, electronic valence, coordination number or the nature of ligands, although some of these can also produce initial state effects. In the case of gallium in hydrated $\mathrm{Ga}_{2} \mathrm{O}_{3}$, where the coordination environment of gallium is expected to be mainly octahedral, we observe the highest modified Auger parameter (1082.8 eV). The modified Auger parameter of the impregnated Ga-solid is $1081.9 \mathrm{eV}$ and this difference compared to bulk $\mathrm{Ga}_{2} \mathrm{O}_{3}$ could be attributed to the occurrence of finely dispersed gallium oxide nanoparticles interacting with the silica surface, as reported by Liu et al.[35]. The modified Auger parameter associated with Ga-37 and Ga-74 solids is clearly lower and distinct from those of Ga-impregnated and bulk $\mathrm{Ga}_{2} \mathrm{O}_{3}$. This lower value of the Auger parameter suggests the presence of tetrahedral $\mathrm{Ga}$ inserted in the silica framework. This interpretation is analogous to the work done by Collard et al. [31]on Zn-based materials. It can be noted that the proximity of the

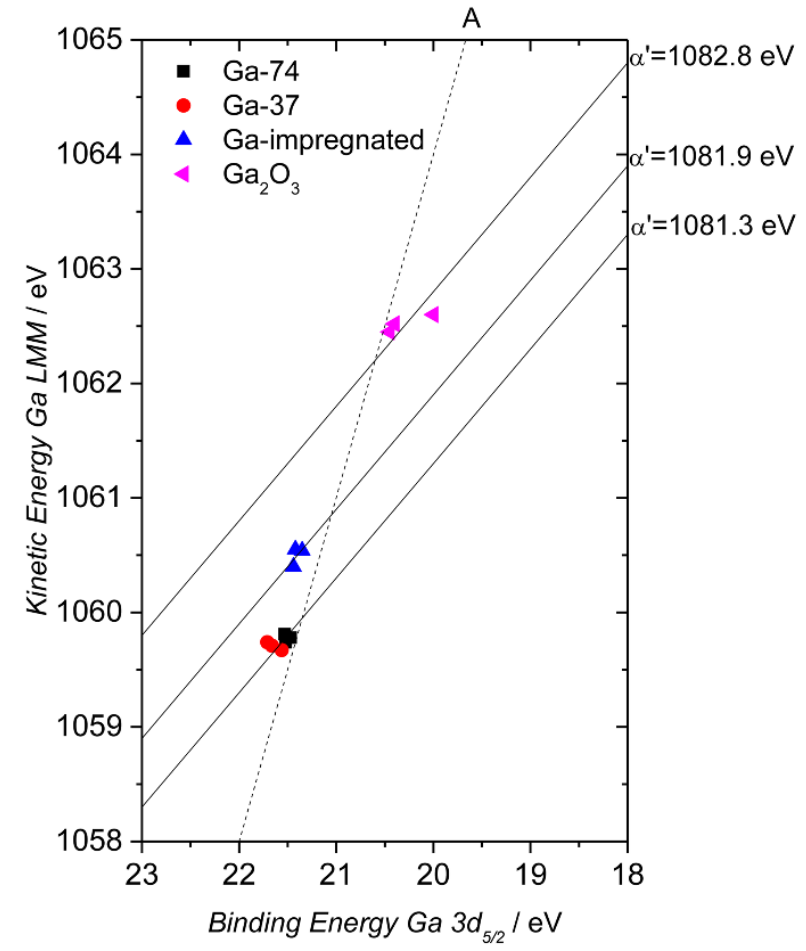

Figure 2. Wagner plot (Ga LMM Auger vs. XPS Ga3d5/2) of Ga-74, Ga-37, Ga-impregnated and gallium oxide 
energy contributions associated with Ga-37 and Ga-74 solids does not allow to appreciate any difference in the chemical environment of gallium in these two materials.

To further investigate the coordination of gallium, solid state ${ }^{71} \mathrm{Ga}$ NMR spectroscopy was exploited. It is known that solid state NMR spectroscopy is a powerful tool to investigate the coordination number/geometry of metal centers inserted as single site within the silica matrix. Gallium has two NMR active nuclei, ${ }^{69} \mathrm{Ga}$ and ${ }^{71} \mathrm{Ga}$, both with a nuclear spin of $3 / 2$ and a natural abundance of 60.1 and 39.8 respectively. It deserves to be mentioned that due to the large second-order quadrupolar broadening, solid state investigation of ${ }^{69 / 71} \mathrm{Ga}$ NMR is challenging. Hence, despite to its lower natural abundance, ${ }^{71} \mathrm{Ga}$ was selected in this work the study due to its smaller quadrupolar moment and slightly higher absolute sensitivity compared to ${ }^{69} \mathrm{Ga}$. In addition to the intrinsic difficulties of ${ }^{71} \mathrm{Ga}$, our materials display an unfavorable combination of features for solid state NMR investigations: the presence of an amorphous skeleton together with a low wt\% of gallium. In order to obtain precise insights on the coordination of gallium, investigations were performed under magic angle spinning (MAS) at $25 \mathrm{kHz}$ at a high magnetic field (11.7 T).

A sample of $\beta-\mathrm{Ga}_{2} \mathrm{O}_{3}$ was first measured as standard compound for our NMR investigation. The corresponding spectrum, in agreement with literature data [40], is reported in Figure S2.

Figure 3 shows the MAS NMR spectra of a hydrated sample of gallium oxide, Ga-impregnated, Ga-148, Ga-74 and Ga-37 respectively. The MAS NMR analysis of hydrated gallium oxide shows the presence of a signal centered at 0 ppm with respect to the reference. This signal is attributed to octahedral gallium [30].

The NMR analysis of Ga-37 revealed the presence of a major contribution centered at $140 \mathrm{ppm}$ with respect to the reference. This contribution has already been observed for other gallo-silicates and is ascribed to tetra-coordinated gallium [26, 27, 30]. Nevertheless, the signal shows also a second contribution centered at $0 \mathrm{ppm}$, which can be associated to the presence of extraframework gallium. These extra-framework species are expected [10] to have an octahedral environment and to be catalytically inactive. The analysis of Ga-74 evidences a narrower contribution centered at $140 \mathrm{ppm}$ and a contribution at $0 \mathrm{ppm}$ which is proportionally less important, as compared to the case of $\mathrm{Ga}-37$. This indicates the presence of a lower proportion of extraframework $\mathrm{Ga}$ in Ga-74. The ${ }^{71} \mathrm{Ga}$ NMR pattern of Ga-148 was similar to Ga-74, suggesting a similar degree of dispersion in both solids. This finding clearly indicates that among the three synthesized solids a Si/Ga ratio of 74 represents the best compromise between Ga loading and isomorphic substitution and highlights the Ga-74 material as the most promising catalyst of the series. As expected (see XPS investigation via Wagner plot), the Ga-impregnated solid displays a combination of intra- and extraframework species which is intermediate between $\mathrm{Ga}_{2} \mathrm{O}_{3}$ and $\mathrm{Ga}-37$. This behavior can be ascribed to the presence of finely dispersed extra-framework species at the surface, some of them in a distorted tetrahedral environment.

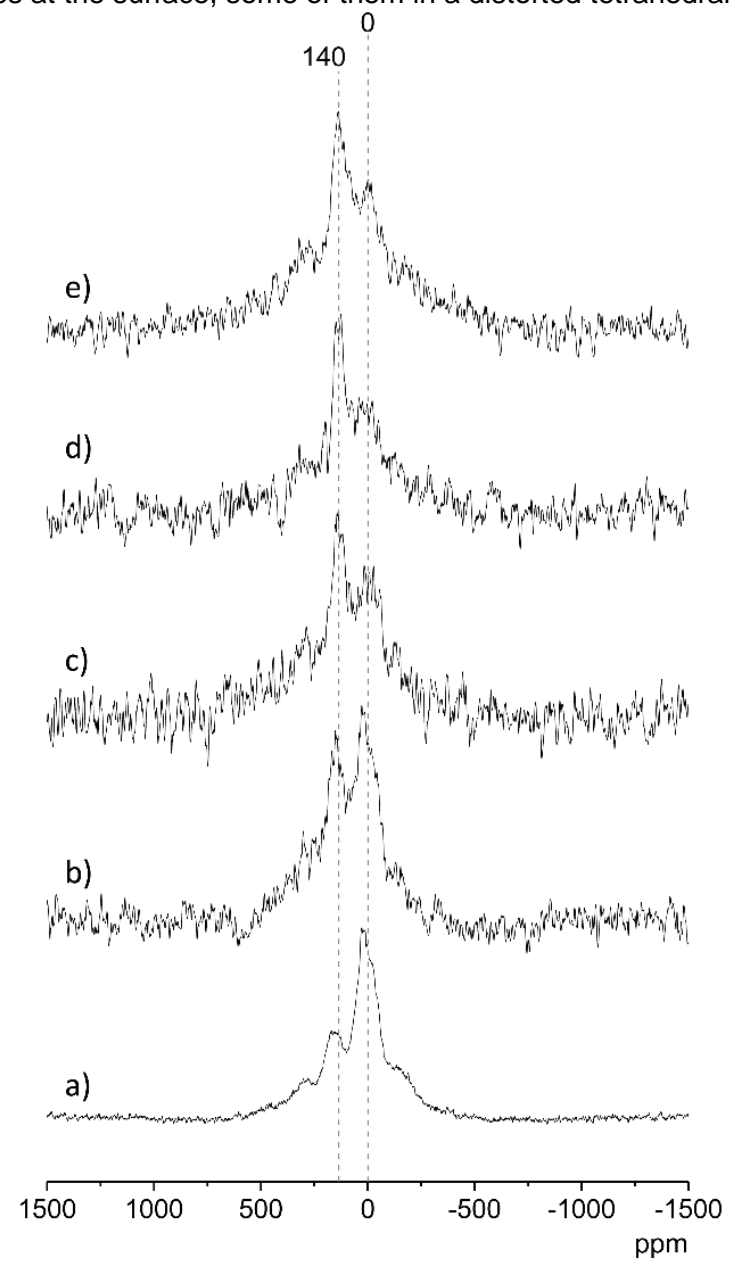

Figure 3. ${ }^{71} \mathrm{Ga}$ solid state MAS NMR spectra of hydrated $\mathrm{Ga}_{2} \mathrm{O}_{3}(\mathrm{a})$, Gaimpregnated (b), Ga-148 (c), Ga-74 (d) and Ga-37 (e) 

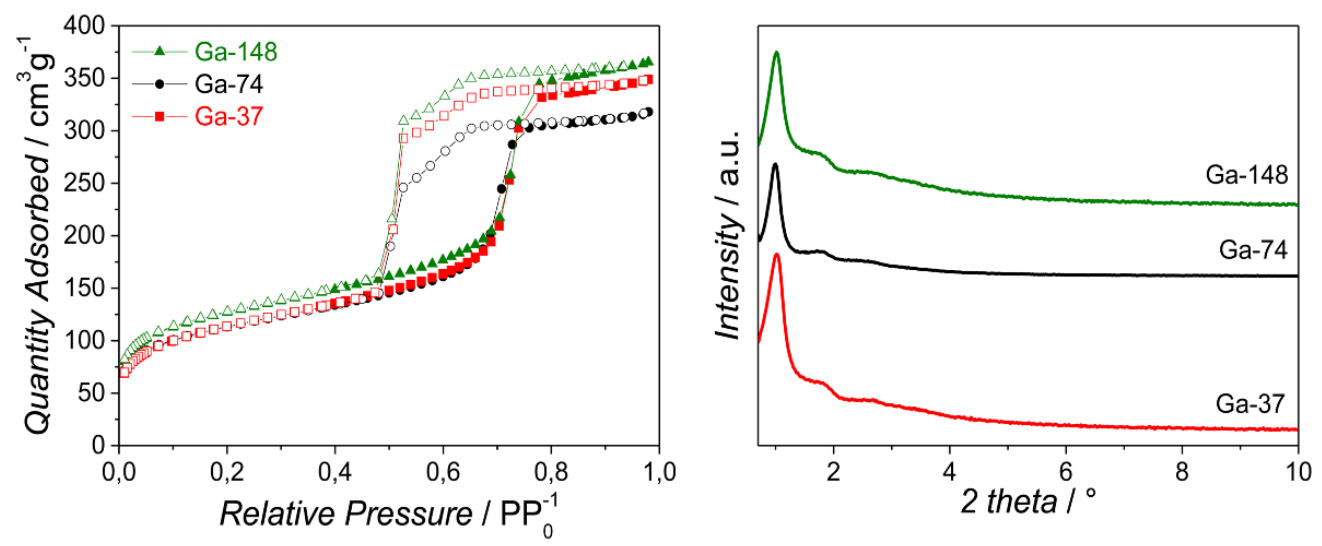

Figure 4. Nitrogen adsorption/desorption isotherms of Ga-silicate solids (left) and small-angle XRD patterns (right)

Table 1. Textural properties and composition of Ga-silicate solids

\begin{tabular}{lcccc}
\hline Material & Surface Area $\left(\mathrm{m}^{2} \cdot \mathrm{g}^{-1}\right)$ & Pore diameter $(\mathrm{nm})$ & Pore Volume $\left(\mathrm{cm}^{3} \cdot \mathrm{g}^{-1}\right)$ & $\mathrm{Si} / \mathrm{Ga}^{[\mathrm{a}]}$ \\
\hline Ga-37 & 350 & 7.5 & 0.5 & 38 \\
Ga-74 & 350 & 7.0 & 0.5 & 79 \\
Ga-148 & 380 & 7.7 & 0.5 & 160 \\
\hline [a] determined via ICP-OES analysis & &
\end{tabular}

The textural properties and the structure of the solids were examined using $\mathrm{N}_{2}$-physisorption and powder XRD. An overview of the textural properties of the synthesized Ga-silicates is reported in Table 1. All solids prepared by aerosol had similar texture with a specific surface area around $350 \mathrm{~m}^{2} \mathrm{~g}^{-1}$ and a pore volume around $0.5 \mathrm{~cm}^{3} \mathrm{~g}^{-1}$. They all showed a type-IV isotherm characterized by an evident hysteresis loop that can be classified as a combination of types $\mathrm{H} 1$ and $\mathrm{H} 2$ (Figure 4, left). The combination of two types of hysteresis may be attributed to the presence of a combination of tubular pores opened at both ends and a more disordered porosity in good accord with TEM observations. The analysis performed using BJH on the adsorption branch of the isotherm showed a narrow pore size distribution centered at around $7.5 \mathrm{~nm}$ for all the solids.

The low angle powder XRD patterns of the materials (Figure 4, right) featured an intense first order $d_{100}$ diffraction peak centered at $2 \theta=1.0^{\circ}$ accounting for mesostructuration of these solids. A second weaker contribution can be observed and is assigned to the $d_{110}$ reflection. From TEM, $\mathrm{N}_{2}$-physisorption and powder XRD investigation, no major differences emerged comparing the morphology and textural properties of the three samples. Therefore, we can argue that raising the concentration of $\mathrm{Ga}$, in the selected range, does not influence the morphology, texture and structure of the solids. Hence, the eventual catalytic differences can be mainly ascribed to different Ga loading and to different insertion/coordination of Ga in the silica structure. S5

The isomorphic substitution of silicon with gallium is known to generate a combination of Lewis and Brønsted acid sites. In order to investigate the acidic properties, the sample Ga-74 has been selected and studied via FT-IR of adsorbed ammonia.

The FT-IR difference spectra $\left(3800-2500 \mathrm{~cm}^{-1}\right.$ and $1800-1300 \mathrm{~cm}^{-1}$ range) of ammonia adsorption on Ga-74 are shown in Figure S3. The absorption band at $1635 \mathrm{~cm}^{-1}$ is assigned to the bending mode of ammonia molecules $\mathrm{H}$-bonded to hydroxyl groups, whereas the broad signal at around $1460 \mathrm{~cm}^{-1}$ is due to the bending mode of $\mathrm{NH}_{4}{ }^{+}$ions generated from proton-transfer reaction on Brønsted acidic groups. This suggests that the incorporation of $\mathrm{Ga}$ within the silica framework is able to generate Brønsted sites acid enough to protonate ammonia. The surface hydroxyls of pure silica materials, on the contrary, interact with $\mathrm{NH}_{3}$ via hydrogen-bonding without proton transfer. The component at around $1620 \mathrm{~cm}^{-1}$, clearly discernable on the sample outgassed

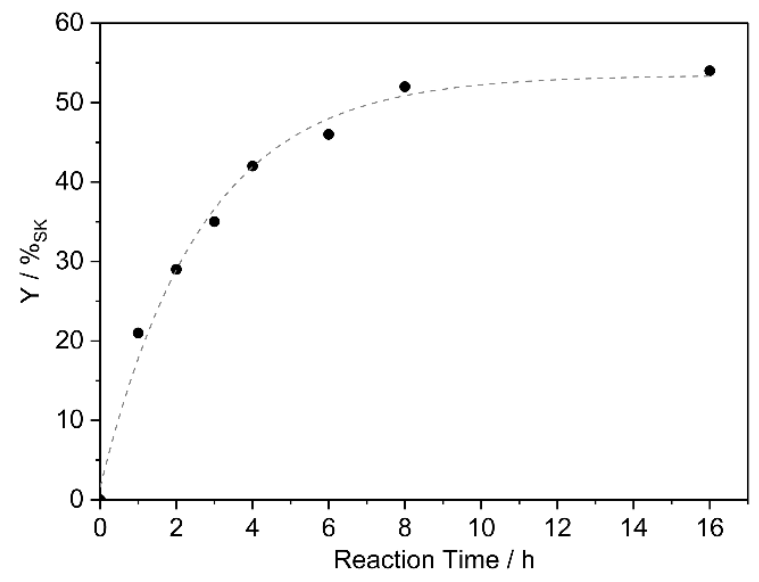

Figure 5. Kinetic study performed using Ga-74 catalyst 
after ammonia adsorption, is ascribed to $\mathrm{NH}_{3}$ molecules adsorbed on unsaturated $\mathrm{Ga}^{3+}$ cations acting as Lewis sites. All the synthesized Ga-silicates present favorable features for their application in heterogeneous catalysis. The targeted reaction selected in this work is represented by the acetalization of glycerol with acetone to produce solketal. A kinetic study was performed using Ga-74 as catalyst and the formation of solketal over time was followed in the range of $1 \mathrm{~h}$ to $16 \mathrm{~h}$, reaching a solketal yield of about $55 \%$ (Figure 5). Extending the reaction time did not result in a further improvement in the conversion. A similar behavior was already observed in literature [41]. This suggests that the thermodynamic equilibrium is reached and that any further improvement in solketal yield could only obtained by the modifications of reaction conditions (e.g. reactants ratio, or product removal) which can shift the equilibrium to the formation of the desired product.

Table 2. Catalytic activity of Ga-silicates in the conversion of glycerol to solketal

\begin{tabular}{cccccc}
\hline Entry & Material & Si/Ga & Yield (\%) & Sel. (\%) & TOF \\
\hline 1 & Ga-37 & 38 & 30 & 85 & 361 \\
2 & Ga-74 & 79 & 28 & 85 & 677 \\
3 & Ga-148 & 160 & 10 & 79 & 487 \\
4 & Ga-impregnated & 37 & $<2$ & - & - \\
5 & $\mathrm{Ga}_{2} \mathrm{O}_{3}$ & - & $<2$ & - & - \\
\hline
\end{tabular}

$\mathrm{Si} / \mathrm{Ga}$ ratios determined by ICP-OES analysis; Reaction conditions: $10 \mathrm{mg}$ of catalyst, $0.01 \mathrm{~mol}$ of glycerol, $0.04 \mathrm{~mol}$ of acetone, $50^{\circ} \mathrm{C}, 2 \mathrm{~h}$; TOF $=\left(n_{\text {solketal }} / n_{G a}\right) \cdot \mathrm{h}^{-1}$

The kinetic study was used to select an adequate reaction time to compare the catalytic activity of the solids. A reaction time of $2 \mathrm{~h}$ was selected as the best compromise between an acceptable solketal yield (around 30\%) enabling an accurate quantification and a meaningful comparison among the catalysts, but far enough from the equilibrium conversion [42]. Thus, the initial specific activity was approached by considering the activity at $2 \mathrm{~h}$, expressed in terms of turnover frequency (TOF, defined as moles of solketal produced per moles of $\mathrm{Ga}$ and per hour). To verify the reproducibility of the catalytic tests, all the solids were tested three times under identical reaction conditions. The error measured on the solketal yield was around $2 \%$, confirming the good reproducibility of the presented tests. From a first comparison among Ga-37, 74 and 148 (Table 2) emerged that the catalytic activity does not increase proportionally with the gallium loading. In particular, very similar solketal yields were observed when a $\mathrm{Si} / \mathrm{Ga}$ ratio of 37 and 74 were used (compare entries 1 and 2, Table 2). The decreased turnover number of the solid Ga-37 compared to Ga-74 can be explained considering the presence of a higher quantity of extra-framework gallium, as revealed by the ${ }^{71} \mathrm{Ga}$ MAS NMR.

Extra-framework gallium species are not active in the selected target reaction, as demonstrated here experimentally with bulk gallium oxide and Ga-impregnated which displayed no catalytic activity (see entries 4 and 5, Table 2). Concerning Ga-148 catalyst, its decreased activity could be attributed to its low Ga loading resulting in the presence of a limited amount of Ga inserted as

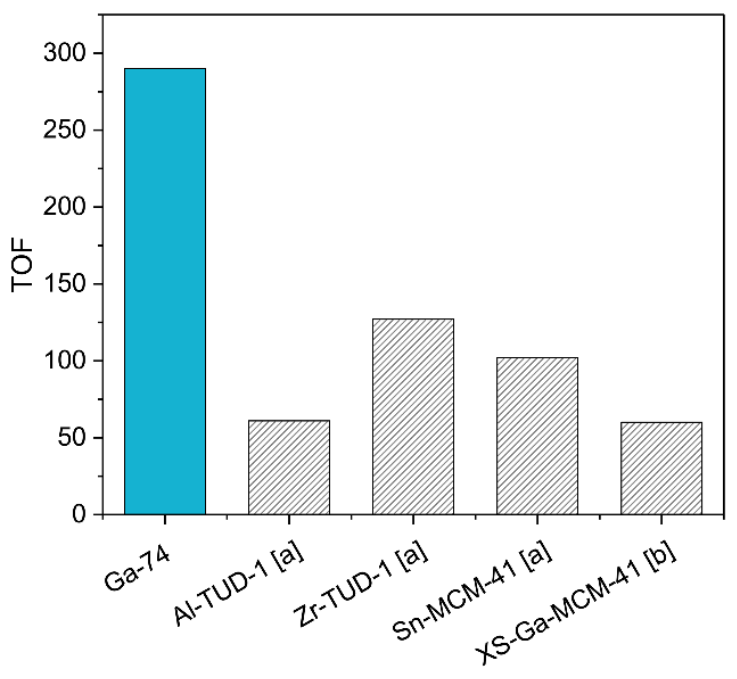

Figure 6. Catalytic activity of Ga-74 in the conversion of glycerol to solketal compared to metal-silicates reported in the literature: [a] ${ }^{[40]}$, $[b]^{[10]}$. Reaction conditions: $0.01 \mathrm{~mol}$ of glycerol, 1 eq. of acetone, $0.02 \mathrm{~mol}$ of t-butanol, $2 \mathrm{~h}$ and $25 \mathrm{mg}$ of catalyst 


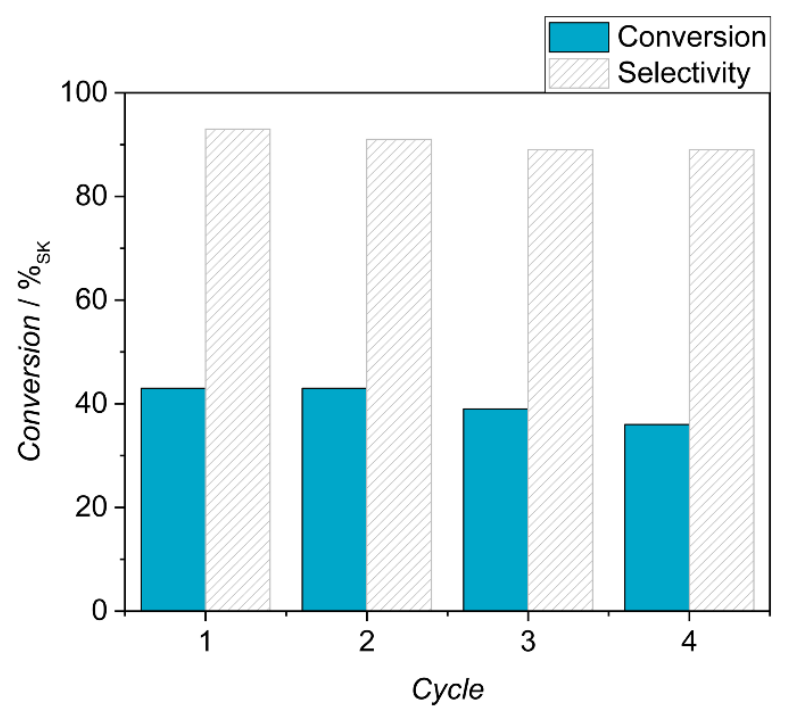

Figure 7. Recycling tests using Ga-74

single sites. All the materials present a very high selectivity and the only secondary product detected was the six-membered ring compounds (2,2-dimethyl-1,3-dioxan-5-ol). The best TOF was obtained employing the Ga-74 catalyst.

While we show that the reaction can be run effectively in solvent-less conditions, several works report the use of solvents for the selected reaction. For this reason, in order to allow a meaningful comparison of our best catalyst with reference solids reported in the literature [10,43], an additional catalytic test was performed, using t-butanol as solvent. The results gathered in Figure 6 evidence the outstanding catalytic performance of Ga-74 compared not only to previously reported Ga-based catalyst but also to other metal-containing silicates reported in literature. This behavior could be due to a favorable combination of features such as high surface area, accessible mesoporosity and insertion of gallium mainly as single site in the silica architecture.

Although the activity of a heterogeneous catalyst is an important parameter, the possibility of using the catalyst in multiple catalytic runs should not be neglected.

Recycling tests for the catalyst showing the best activity (Ga-74) are presented in Figure 7. In order to prove the reusability of the catalyst, four consecutive catalytic tests were performed, including reactivation of the catalyst by washing with acetonitrile and calcination at $450^{\circ} \mathrm{C}$ for two hours (see experimental section). Recycling tests showed that Ga-74 catalyst always maintained an excellent selectivity and an almost stable catalytic activity over four cycles. The absence of leaching of gallium species in solution was verified by ICP-OES analysis on the used catalyst. At the end of the last cycle, no change was observed in term of Si/Ga ratio between the fresh and reused catalyst. In addition, the characterization of the catalyst after recycle via $\mathrm{N}_{2}$ physisorption and ${ }^{71} \mathrm{Ga}$ NMR did not display major differences in comparison to the fresh solid (see Figure S4 and Table S1).

In order to investigate the stability of the active sites under the selected reaction conditions, a hot filtration test was performed as well (Figure S5, see experimental section for reaction conditions). The solketal yield was quantified after one hour of reaction. The catalyst was then separated from the reaction medium by hot filtration $\left(50^{\circ} \mathrm{C}\right)$ and the filtrate was further reacted at $50^{\circ} \mathrm{C}$ for additional five hours. Figure S5 shows that the solketal yield remained unchanged, indicating the absence of leaching of active sites.

\section{Conclusions}

The aerosol process has been used here for the first time as a straightforward synthetic procedure for the synthesis of Ga-silicate catalysts. The solids were fully characterized via transmission electron microscopy, ICP-OES analysis, and $\mathrm{N}_{2}$ physisorption measurements. The catalysts can be described as spherical gallosilicate particles with well-calibrated mesopores, synthesized with an excellent control on the composition. An advanced XPS analysis using the Wagner plot and ${ }^{71} \mathrm{Ga}$ solid state MAS NMR spectroscopy allowed to deeply investigate the coordination environment of Ga. Importantly, this allowed to evidence the successful isomorphic substitution of $\mathrm{Ga}$ in the silica matrix. The best material, Ga-74, exhibited outstanding catalytic activity in the direct conversion of glycerol to solketal under solventless conditions. This material clearly outcompetes previously reported Ga-based catalysts. This high performance was ascribed to the efficient insertion of gallium as single site within the silica framework. Moreover, the catalyst proved to be stable under the selected reaction conditions and reusable in multiple runs. The stability of the catalyst was also supported via full characterization of the solid after the $4^{\text {th }}$ cycle where no major differences were found.

\section{Acknowledgements}

Authors acknowledge the 'Communauté française de Belgique' - including the PhD fellowship of A. Vivian - through the ARC programme (15/20-069) and the FNRS through the FNRS-EQP-U.N034.17F for the financial support. This research used 
resources of the "Plateforme Technologique Physico-Chemical Characterization" - PC ${ }^{2}$, the SIAM platform (Synthesis, Irradiation \& Analysis of Materials) and the MORPH-IM platform (Morphology \& Imaging) located at the University of Namur.

\section{References}

[1] M.E. Davis, Ordered porous materials for emerging applications, Nature, 417 (2002) 813-821.

[2] Y. Li, J. Shi, Hollow-Structured Mesoporous Materials: Chemical Synthesis, Functionalization and Applications, Advanced Materials, 26 (2014) $3176-3205$. [3] B. Jarry, F. Launay, J.P. Nogier, V. Montouillout, L. Gengembre, J.L. Bonardet, Characterisation, acidity and catalytic activity of Ga-SBA-15 materials prepared following different synthesis procedures, Applied Catalysis A: General, 309 (2006) 177-186.

[4] Z. El Berrichi, B. Louis, J.P. Tessonnier, O. Ersen, L. Cherif, M.J. Ledoux, C. Pham-Huu, One-pot synthesis of Ga-SBA-15: Activity comparison with Gapost-treated SBA-15 catalysts, Applied Catalysis A: General, 316 (2007) 219-225.

[5] R. Fricke, H. Kosslick, G. Lischke, M. Richter, Incorporation of Gallium into Zeolites: Syntheses, Properties and Catalytic Application, Chemical Reviews, $100(2000)$ 2303-2406.

[6] M.L. Occelli, G. Schewering, C. Fild, H. Eckert, A. Auroux, P. Iyer, Galliosilicate molecular sieves with the faujasite structure, Microporous and Mesoporous Materials, $34(2000) 15-22$

[7] G. Giannetto, R. Monque, R. Galiasso, Transformation of LPG into Aromatic Hydrocarbons and Hydrogen over Zeolite Catalysts, Catalysis Reviews, 36 (1994) 271-304

[8] T. Inui, H. Nagata, H. Matsuda, J.B. Kim, Y. Ishihara, Selective synthesis of aromatic hydrocarbons from propylene on gallium-silicate catalysts, Industrial \& Engineering Chemistry Research, 31 (1992) 995-999.

[9] A.P. Singh, K.R. Reddy, Synthesis, characterization, and catalytic activity of gallosilicate analogs of zeolite ZSM-22, Zeolites, 14 (1994) 290-294

[10] X. Collard, L. Li, W. Lueangchaichaweng, A. Bertrand, C. Aprile, P.P. Pescarmona, Ga-MCM-41 nanoparticles: Synthesis and application of versatile heterogeneous catalysts, Catalysis Today, 235 (2014) 184-192.

[11] P.Y. Dapsens, B.T. Kusema, C. Mondelli, J. Pérez-Ramírez, Gallium-modified zeolites for the selective conversion of bio-based dihydroxyacetone into C1-C4 alkyl lactates, Journal of Molecular Catalysis A: Chemical, 388-389 (2014) 141-147.

[12] D.P. Debecker, S. Le Bras, C. Boissière, A. Chaumonnot, C. Sanchez, Aerosol processing: a wind of innovation in the field of advanced heterogeneous catalysts, Chemical Society Reviews, 47 (2018) 4112-4155.

[13] A. Sachse, V. Hulea, K.L. Kostov, N. Marcotte, M.Y. Boltoeva, E. Belamie, B. Alonso, Efficient mesoporous silica-titania catalysts from colloidal selfassembly, Chemical Communications, 48 (2012) 10648-10650.

[14] K.Y. Jung, Y.R. Jung, J.-K. Jeon, J.H. Kim, Y.-K. Park, S. Kim, Preparation of mesoporous V2O5/TiO2 via spray pyrolysis and its application to the catalytic conversion of 1, 2-dichlorobenzene, Journal of Industrial and Engineering Chemistry, 17 (2011) 144-148.

[15] A. Kim, C. Sanchez, B. Haye, C. Boissière, C. Sassoye, D.P. Debecker, Mesoporous TiO2 Support Materials for Ru-Based CO2 Methanation Catalysts, ACS Applied Nano Materials, 2 (2019) 3220-3230.

[16] F. Colbeau-Justin, C. Boissière, A. Chaumonnot, A. Bonduelle, C. Sanchez, Aerosol Route to Highly Efficient (Co)Mo/SiO2 Mesoporous Catalysts, Advanced Functional Materials, 24 (2014) 233-239.

[17] C. Boissiere, D. Grosso, A. Chaumonnot, L. Nicole, C. Sanchez, Aerosol Route to Functional Nanostructured Inorganic and Hybrid Porous Materials, Advanced Materials, 23 (2011) 599-623.

[18] D.P. Debecker, Innovative Sol-Gel Routes for the Bottom-Up Preparation of Heterogeneous Catalysts, The Chemical Record, 18 (2018) $662-675$.

[19] D.P. Debecker, M. Stoyanova, U. Rodemerck, F. Colbeau-Justin, C. Boissère, A. Chaumonnot, A. Bonduelle, C. Sanchez, Aerosol route to nanostructured WO3-SiO2-Al2O3 metathesis catalysts: Toward higher propene yield, Applied Catalysis A: General, 470 (2014) 458-466.

[20] N. Godard, A. Vivian, L. Fusaro, L. Cannavicci, C. Aprile, D.P. Debecker, High-Yield Synthesis of Ethyl Lactate with Mesoporous Tin Silicate Catalysts Prepared by an Aerosol-Assisted Sol-Gel Process, ChemCatChem, 9 (2017) 2211-2218.

[21] V. Smeets, C. Boissière, C. Sanchez, E.M. Gaigneaux, E. Peeters, B.F. Sels, M. Dusselier, D.P. Debecker, Aerosol Route to TiO2-SiO2 Catalysts with Tailored Pore Architecture and High Epoxidation Activity, Chemistry of Materials, 31 (2019) 1610-1619.

[22] Z. Guo, G. Xiong, L. Liu, P. Li, L. Hao, Y. Cao, F. Tian, Aerosol-assisted synthesis of hierarchical porous titanosilicate molecular sieve as catalysts for cyclohexene epoxidation, Journal of Porous Materials, 23 (2016) 407-413.

[23] S. Maksasithorn, P. Praserthdam, K. Suriye, D.P. Debecker, Preparation of super-microporous WO3-SiO2 olefin metathesis catalysts by the aerosolassisted sol-gel process, Microporous and Mesoporous Materials, 213 (2015) 125-133.

[24] S. Pega, C. Boissière, D. Grosso, T. Azaïs, A. Chaumonnot, C. Sanchez, Direct Aerosol Synthesis of Large-Pore Amorphous Mesostructured Aluminosilicates with Superior Acid-Catalytic Properties, Angewandte Chemie International Edition, 48 (2009) $2784-2787$.

[25] A. Vivian, L. Fusaro, D.P. Debecker, C. Aprile, Mesoporous Methyl-Functionalized Sn-Silicates Generated by the Aerosol Process for the Sustainable Production of Ethyl Lactate, ACS Sustainable Chemistry \& Engineering, 6 (2018) 14095-14103.

[26] C.R. Bayense, A.P.M. Kentgens, J.W. De Haan, L.J.M. Van de Ven, J.H.C. Van Hooff, Determination of gallium in H(Ga)ZSM5 zeolites by gallium-71 MAS NMR spectroscopy, The Journal of Physical Chemistry, 96 (1992) 775-782.

[27] M. Chatterjee, T. Iwasaki, Y. Onodera, T. Nagase, H. Hayashi, T. Ebina, Characterization of Ordered Mesoporous Gallium MCM-41 Synthesized at Room Temperature, Chemistry of Materials, 12 (2000) 1654-1659.

[28] C.-F. Cheng, H. He, W. Zhou, J. Klinowski, J.A.S. Gonçalves, L.F. Gladden, Synthesis and Characterization of the Gallosilicate Mesoporous Molecular Sieve MCM-41, The Journal of Physical Chemistry, 100 (1996) 390-396.

[29] W. Zhang, C.I. Ratcliffe, I.L. Moudrakovski, J.S. Tse, C.-Y. Mou, J.A. Ripmeester, Characterization of the location and interfacial states of gallium in gallium/MCM-41 composites, Microporous and Mesoporous Materials, 79 (2005) 195-203.

[30] H.K.C. Timken, E. Oldfield, Solid-state gallium-69 and gallium-71 nuclear magnetic resonance spectroscopic studies of gallium analog zeolites and related systems, Journal of the American Chemical Society, 109 (1987) 7669-7673.

[31] X. Collard, P. Louette, S. Fiorilli, C. Aprile, High surface area zincosilicates as efficient catalysts for the synthesis of ethyl lactate: an in-depth structural investigation, Physical Chemistry Chemical Physics, 17 (2015) 26756-26765.

[32] J.L. Bourque, M.C. Biesinger, K.M. Baines, Chemical state determination of molecular gallium compounds using XPS, Dalton Transactions, 45 (2016) 7678-7696.

[33] C.D. Wagner, A. Joshi, The auger parameter, its utility and advantages: a review, Journal of Electron Spectroscopy and Related Phenomena, 47 (1988) 283-313.

[34] R. Gérardy, R. Morodo, J. Estager, P. Luis, D.P. Debecker, J.-C.M. Monbaliu, Sustaining the Transition from a Petrobased to a Biobased Chemical Industry with Flow Chemistry, Topics in Current Chemistry, 377 (2018) 1.

[35] R.-I. Liu, H.-q. Zhu, Z.-w. Wu, Z.-f. Qin, W.-b. Fan, J.-g. Wang, Aromatization of propane over Ga-modified ZSM-5 catalysts, Journal of Fuel Chemistry and Technology, 43 (2015) 961-969.

[36] G. Moretti, Auger parameter and Wagner plot in the characterization of chemical states by X-ray photoelectron spectroscopy: a review, Journal of electron spectroscopy and related phenomena, 95 (1998) 95-144.

[37] G. Moretti, F. Filippone, M. Satta, Use of Auger parameter and Wagner plot in the characterization of Cu-ZSM-5 catalysts, Surface and Interface Analysis: An International Journal devoted to the development and application of techniques for the analysis of surfaces, interfaces and thin films, 31 (2001) $249-254$. [38] C. Wagner, A. Joshi, The auger parameter, its utility and advantages: a review, Journal of Electron Spectroscopy and Related Phenomena, 47 (1988) 283-313.

[39] G. Moretti, A. Palma, E. Paparazzo, M. Satta, Auger parameter and Wagner plot studies of small copper clusters, Surface Science, 646 (2016) 298-305. [40] D. Massiot, I. Farnan, N. Gautier, D. Trumeau, A. Trokiner, J.P. Coutures, 71Ga and 69Ga nuclear magnetic resonance study of $\beta$-Ga2O3: resolution of four- and six-fold coordinated Ga sites in static conditions, Solid State Nuclear Magnetic Resonance, 4 (1995) $241-248$.

[41] L.A. Bivona, A. Vivian, L. Fusaro, S. Fiorilli, C. Aprile, Design and catalytic applications of 1D tubular nanostructures: Improving efficiency in glycerol conversion, Applied Catalysis B: Environmental, 247 (2019) 182-190.

[42] F. Schüth, M.D. Ward, J.M. Buriak, Common Pitfalls of Catalysis Manuscripts Submitted to Chemistry of Materials, Chemistry of Materials, 30 (2018) 3599-3600

[43] L. Li, T.I. Korányi, B.F. Sels, P.P. Pescarmona, Highly-efficient conversion of glycerol to solketal over heterogeneous Lewis acid catalysts, Green Chemistry, 14 (2012) 1611-1619. 
